\title{
Research of Localization Algorithm Based on Multi-QR Code Yong-feng Huang ${ }^{1, a}$, Zhen Xue ${ }^{1, b,{ }^{*}}$, Cai-rong Yan $^{1, c}$ \\ ${ }^{1}$ School of Donghua University, Shanghai 201620, China; \\ a yfhuang@mail.dhu.edu.cn, b13262790360@163.com, c cryan@ dhu.edu.cn
}

Keywords: QR code,multi-QR code recognition,position detection pattern,center points grouping

\begin{abstract}
Location - aware Service based on QR code is widely used in the large complex indoor environment because of its strong anti-interference ability, wide coverage and high positioning accuracy.For the situation that the QR code position sensing process may exist multi-QR code, an multi-QR code localization algorithm is proposed. We use QR code module size,version number and other characteristics of the code information to group these position detection pattern center points, which based on the existing QR code detection center point detection algorithm and can be proved to identify multi-QR code.In the case,the recognition rate is about $94 \%$.
\end{abstract}

\section{Introduction}

With the accelerating of urbanization, the internal structure of large-scale buildings is becoming more complex, which has brought a serious challenge to people's perception of space, especially in field of the indoor environment such as in shopping malls, airports and convention center.Indoor navigation systems and applications gradually rising recently. But in a complex indoor environment, Bluetooth and WIFI exist some shortcomings. The application process is limited because of the shortcomings about Bluetooth and WIFI's low stability and height error.The technology of indoor positioning with visual markers has become a research hotspot of indoor location technology because of its low cost, wide coverage, high precision and strong robustness. The QR code is one of the most common visual markers[1].

QR code records the data by using black and white graphics distributed in a square with some rules[2]. When the existing QR code recognition algorithm identify the QR code,firstly,you need to determine the location of center of graphics about QR code position detection[3,4].Then,you should record the number of center points that you find.If the points are not three,you can affirm that this figure is not QR code.If the points are three,we can determine the QR code's parameter such as version number, positioning graphics, error correction and coding area,and acquire QR code storage of other information.However, in the process of locating the center point information about the position detection, some wrong points may be regarded as the center point, which will lead to the recognition failure in the subsequent operation.

In addition, in the large-scale complex indoor environment, at the same time, the same camera sometimes there will be multi-QR code.If based on the traditional single-QR code algorithm,we can not identify multi-QR code effectively when the center points number are above three. In order to identify multi-QR code at the same time, based on the general QR code identification algorithm is proposed on the basis of multi-QR code localization algorithm. First, make sure all could be a QR code in the image position detection center of graphic information. Secondly, according to whether can find positioning graphics as the basis of center group. Finally, according to the result of grouping to decode the QR code. The experimental results show that the algorithm not only good effect under the single QR code, and can satisfy the requirements under the QR code more scenes.

\section{Multi-QR Code Localization Algorithm}

The Localization algorithm is how to determine the position of the QR code in image.A QR code consists of three position detection pattern. The three position detection patterns have the same shape and size, and are distributed at the three corners of the symbol pattern. No matter which point of 
view,the position structure both the dark and light module width ratios are 1:1:3:1:1[5]. Therefore, when locating the QR code symbols, it is possible to locate the QR code symbols as long as the three detection patterns can be positioned.

In the case of multi-QR code recognition, the center points of position detection pattern is must be above 3.Under the situation, it is particularly important to correctly group the center points, since the number of the detected position detection pattern center points must be greater than three, and the wrong center point combination leads to recognition failure. In order to solve the above problem, this paper proposes a grouping algorithm of center point of position detection pattern (see Algorithm 1).From the characteristics of QR code, we can know the number of center points in each group is 3, that is, all the cases were divided into $C_{n}^{3}$ groups,among them,n is the number of detected center points. First, it is determined whether there is a dead pixel(the point satisfying the fixed module ratio but not the center point) in the center point obtained by the definition 3 and removing the dead pixel.Next,we know the relationship between the QR code version number and the module size. For a fixed version of the QR code,the distance between the center point is a fixed value. Because the certain error about QR code module size, the center points which satisfy the distance between the two center points in the range of $7 \times \mathrm{M} \sim 21 \times \mathrm{M}$ are divided into the same group.Finally, the other center points are grouped according to the above rule until all the elements of the center points detected into a grouped collection.

Algorithm 1 Position detection center points grouping algorithm

Input: The original image matrix after binarization $F$, Center collection $D=\{D 1, D 2, \ldots, D k\}$

Center of the scale $\mathrm{M}=\{\mathrm{M} 1, \mathrm{M} 2, \ldots, \mathrm{Mk}\}$, $\mathrm{k}$ as the center of the detected number among them

Output: The center of the group set correctly $\mathrm{E}=\{\mathrm{E} 1, \mathrm{E} 2, \ldots, \mathrm{Eq}\}$,q is the code number
(1) Calculate the value of the $\mathrm{z}=\mathrm{k} \% 3$
(2) According to the center of size $\mathrm{M}$ calculation variance,remove the largest $\mathrm{z}$ center from the set of center.
(3) In turn to take out the center $\mathrm{Dk}$, computing center with the remaining distance size $\mathrm{H}$
(4) If $\mathrm{H}$ within may become a QR code size and number is 3,then these center are ssigned to group sequence.
(5) The cycle will not end until all center into the grouped sequences,then we can get center set $\mathrm{E}$ after grouping

\section{Experiments}

In this paper, the experimental main purpose is to obtain the recognition rate of the improved QR recognition which is using the multi-QR code localization algorithm. In order to study the influence of the correct recognition rate of this algorithm under different scenarios, the experiment adopts size is $15 \mathrm{~cm} \times 15 \mathrm{~cm}$ QR code. In addition, QR code position is not a good judge QR code identification efficiency, this article adopts the strategy of randomized experiment.

According to different application scenarios, this paper collected a lot of video about QR code, and extract each frame to carry on the experiment under the different scenarios. Through analyzing application scenario, the influence factors of the experimental results are recognition of distance, Angle, light conditions, background and complex conditions appear fuzzy circumstance in the process of collecting video frames. This article does a large number of experiment in the school library, school buildings and other large indoor environment.The experiment established a image database of 1000 video frame, and classified according to the influencing factors on the experimental data, the classification is shown in Table 1.

Table1 Factors affecting the classification

\begin{tabular}{|c|c|c|}
\hline Influence factor & Experimental data & Note \\
\hline distance & 400 & $1 \mathrm{~m} / 2 \mathrm{~m} / 3 \mathrm{~m} / 4 \mathrm{~m}$ \\
\hline angle & 200 & $45^{0} / 60^{0} / 90^{0} / 120^{0} / 135^{0}$ \\
\hline light & 1000 & light $/$ sunlight \\
\hline background & 600 & white/other objects \\
\hline fuzzy & 50 & presents the fuzzy \\
\hline
\end{tabular}


Among them, the experiment shows the size of $15 \mathrm{~cm} \times 15 \mathrm{~cm}$ QR code identification distance is greater than $1 \mathrm{~m}$, less than $5 \mathrm{~m}$. Therefore, this article will identify distance experiment is set to $1 \mathrm{~m} /$ $2 \mathrm{~m} / 3 \mathrm{~m} / 4 \mathrm{~m}$. In the Angle of the influence factors for identifying experiments, this algorithm will define the QR code identification frame data as "positive", other conditions are all "oblique". Light consists of light and sunlight. In the case of poor weather conditions, this paper experiment indoors only under the condition of light. Other conditions are conducted in the sun. For other objects exist on the efficiency of the algorithm to identify, in this paper, by setting the different background, white simple situation as background, the other for complex background. Finally extract in fuzzy frame that exist in the experimental process separately experiment, investigation in this paper, algorithm under the condition of fuzzy recognition rate size.The experiment database example shown in Fig.1.

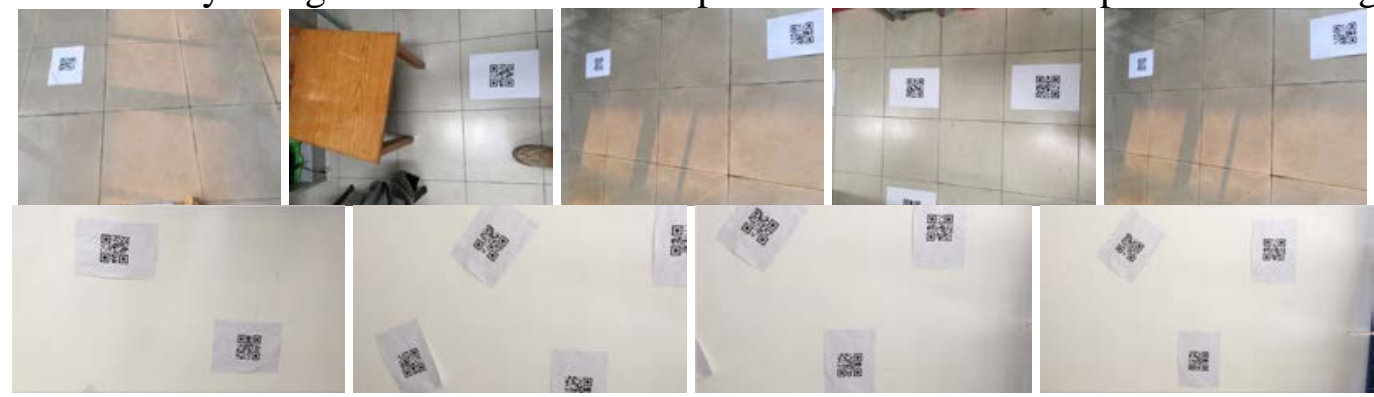

Fig.1 The experiment database example

Because each influence factor is applied to identify common efficiency, so on the analysis of each kind of factor influence on the result of the experiment, the other factors as fixed. According to common sense, in the light, the background is white, the image is not under the condition of fuzzy recognition rate are higher than other cases. As a result, some factors influence on the result of the experiment, other factors limit is relatively simple. Various factors influence on the recognition efficiency as shown in Fig. 2.
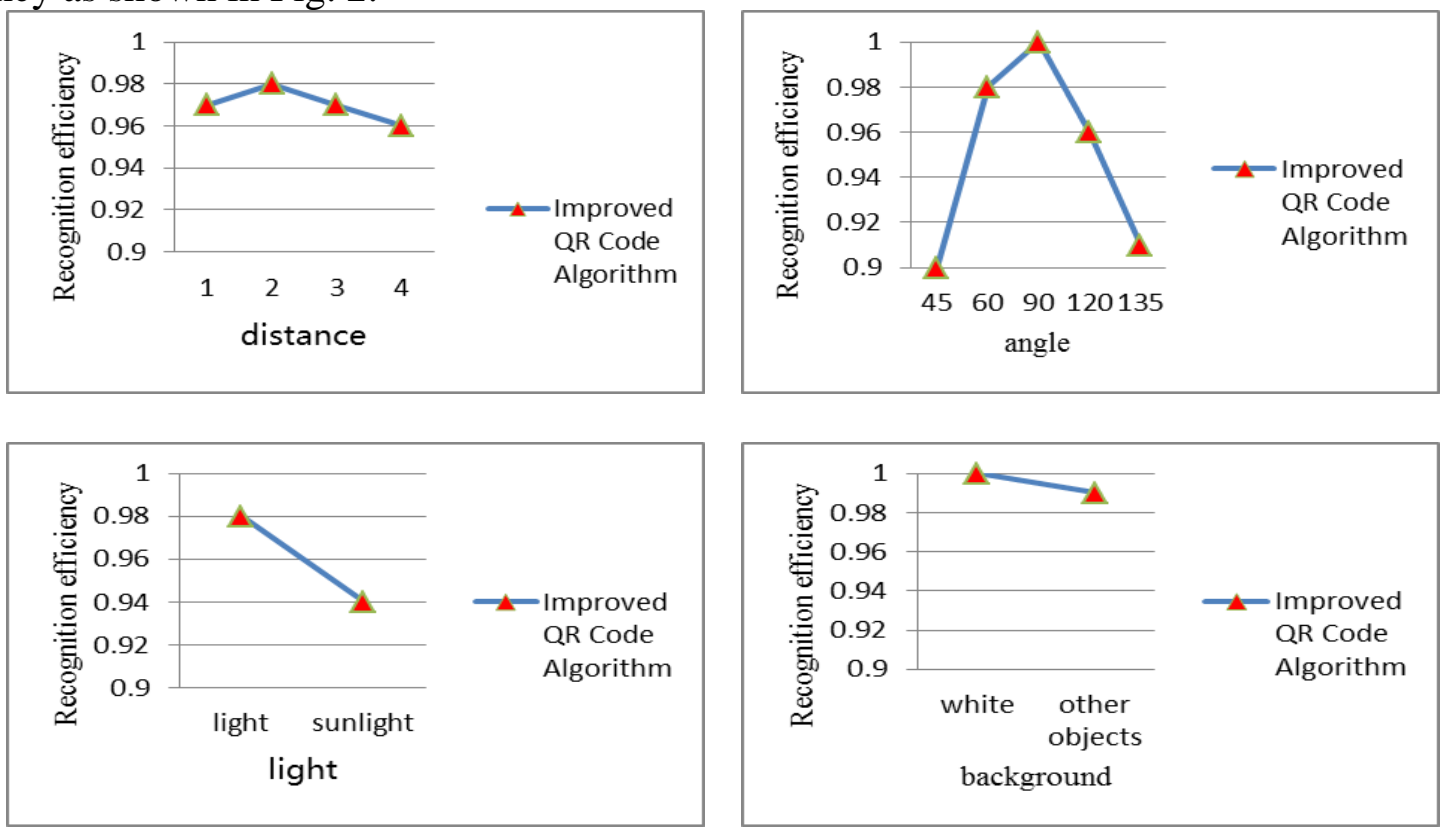

Fig. 2 Various factors influence on the recognition efficiency

In addition, after 50 group experiment indicated in fuzzy frame cases the improved QR code identification efficiency of $60 \%$. For the proposed in large complex in the indoor navigation and positioning of QR code,through experiment, found many QR code only related to the identification distance, identify more QR code appears in relation to the distance as shown in Table 2. 
Table 2 Three Scheme comparing

\begin{tabular}{|c|c|c|}
\hline Distance & Amount & Muti-QR code \\
\hline 1 & 100 & 0 \\
\hline 2 & 100 & 50 \\
\hline 3 & 100 & 90 \\
\hline 4 & 100 & 100 \\
\hline
\end{tabular}

Comprehensive experiment and the result above, this article put forward the improvement of QR code recognition algorithm are greatly influenced by identifying Angle, lighting conditions, and identify the distance is less affected with the condition of complicated background, the identification of overall efficiency of $94 \%$.

\section{Conclusions}

This paper proposes an improved QR code recognition algorithm which is using multi-QR code localization algorithm.We will group the center point of position detection pattern correctly using QR code version number,module size,relations between center distance which based on the original QR code position detection pattern center points detection algorithm,By this method ,we can identify multi-QR code. Analysis for algorithm and expreiments show that the algorithm has good recognition efficiency under the condition of multi-QR code.

\section{References}

[1] Lin D T, Lin C L. Automatic location for multi-symbology and multiple 1D and 2D barcodes[J]. Journal of Marine Science \& Technology, 2013, 21(6):663-668.

[2] Chu C H, Yang D N, Pan Y L, et al. Stabilization and extraction of 2D barcodes for camera phones[J]. Multimedia Systems, 2011, 17(2):113-133.

[3] Zou X, Liu G D, Wang J M. Study on the sequence of steps in the QR code image preprocessing[C]// International Conference on Future Computer and Communication. IEEE, 2010:V3-446-V3-449.

[4] Liu Q, Li X, Zou M, et al. The multi-QR codes extraction method in illegible image based on contour tracing[J]. 2011:51-56.

[5] Ouldbarikalla E, Sawaya W, Delignon Y, et al. New algorithm for 2D barcode detection[C]// International Conference on Advanced Communication Technology. IEEE Press, 2009:1979-1981. 